Eur. J. Clin. Chem. Clin. Biochem.

Vol. 29, 1991, pp. 277-279

(C) 1991 Walter de Gruyter \& Co.

Berlin - New York

\title{
Reference Methods for Total Cholesterol and Total Glycerol ${ }^{1}$ )
}

\author{
By L. Siekmann \\ Institut für Klinische Biochemie der Universität Bonn, Bonn, Germany
}

(Received January 24, 1991)

Summary: In internal and external quality assessment of clinical chemical analyses, the use of reference method values is prescribed in the Federal Republic of Germany as a measure of accuracy control. In the field of lipid analyses, reference methods are available for measuring total cholesterol and total glycerol in control sera. The reference measurement procedures are based on the highly accurate analytical principle of isotope dilution mass spectrometry. A very detailed protocol that ensures a high level of reliability must be followed when reference measurement procedures are carried out. The introduction of reference methods as a basis for accuracy control has greatly limited the unacceptable use of inaccurate routine methods.

\section{Introduction}

In 1989 a system of reference measurement procedures was introduced in the Federal Republic of Germany for external quality assessment of clinical chemical testing. Reference methods now provide the basis for the evaluation of participants' results in collaborative surveys. This system has its legal basis in the guidelines of the Bundesärztekammer (Federal Medical Association) (1), which refer back to the calibration act. It is now mandatory for clinical chemical laboratories to participate in this quality assurance system, and laboratories which do not fulfil the requirements for a certain clinical chemical analyte will run into trouble with public health insurance. Because of these restrictive regulations and their consequences for all the laboratories, those who organize the collaborative surveys take on a high level of responsibility when they set up reference method values.

Since about 1970, our laboratory has been developing reference measurement procedures on the basis of the methodological principle of isotope dilution mass spectrometry. Twelve different quantities are now reg-

\footnotetext{
1) Based on a lecture given at the Symposium "Reference Methods in Clinical Chemistry - Objectives, Trends, Problems" of the Congress Biochemische Analytik 90, München, May 8, 1990
}

ularly measured in various control sera for internal and external quality assessment. In addition to some clinically interesting hormones and the nitrogen-containing metabolites, creatinine and uric acid, the list of analytes also includes the two lipids, total cholesterol and total glycerol.

\section{Experimental}

The analytical results for "total" cholesterol comprise the fractions of the free and the esterified steroid. In order to measure total cholesterol, ${ }^{13} \mathrm{C}_{2}$-labelled cholesterol is added to an amount of serum which is assumed to contain about $40 \mu \mathrm{g}$ cholesterol. The esterified cholesterol fraction is hydrolysed with ethanolic potassium hydroxide solution, and after diluting with water the steroids are extracted with cyclohexane. Then an aliquot of the organic solvent is evaporated to dryness and the residue is reacted to form the trimethylsilyl ether derivative. A portion of the reaction mixture is injected into a gas liquid chromatography capillary column, the end of which is connected to the electron impact ion source of a quadrupole mass spectrometer. The instrument is tuned to monitor the molecular ions of the cholesterol and the labelled cholesterol derivative continuously at $\mathrm{m} / \mathrm{z} 458$ and $\mathrm{m} / \mathrm{z} 460$. Using the selected ion mon- 
itoring technique, the ratio of the non-labelled and the labelled cholesterol can be determined. Parallel to this, a series of standard mixtures is analysed containing defined amounts of cholesterol reference material from the National Institute of Standards and Technology (NIST) and the same amount of the labelled cholesterol that has been added to the serum samples. The analytical results are calculated from the isotope ratios measured in the serum samples and the standard mixtures.

For the analysis of "total glycerol", serum samples are hydrolysed with ethanolic potassium hydroxide solution in the presence of ${ }^{13} \mathrm{C}_{2}$-glycerol. The serum proteins are precipitated by addition of absolute ethanol and centrifugation. Supernatant $(1 \mathrm{ml})$ is evaporated to dryness and reacted with heptafluorobutyric anhydride in acetonitrile. A portion of the reaction mixture is analysed by gas chromatography mass spectrometry, using a fused silica capillary column, $30 \mathrm{~m}$ long and coated with SE-52, and leading the column eluate into the electron impact ion source of the quadrupole mass spectrometer. The glycerol derivative is monitored at $\mathrm{m} / \mathrm{z} 467$ and the corresponding labelled glycerol at $\mathrm{m} / \mathrm{z} 469$.

The analytical results are calculated from the isotope ratios measured in samples and standard mixtures. Tripalmitin, obtained from the NIST, is used as a primary reference material.
The "total glycerol" determined by this method is made up of the same fractions of free glycerol and the glycerol fatty acid esters as measured by the commercial test kits. The phospholipids, which are not hydrolysable with ethanolic potassium hydroxide solution, do not contribute to the glycerol concentration determined by the so-called "tri-glyceride" test kits or the reference method as described here.

\section{Results}

The high specificity of the mass spectrometric detection procedure may be demonstrated by the two chromatograms recorded simultaneously for the labelled and the non-labelled cholesterol derivative shown in figure 1. In addition to the two substances of interest, no additional peaks can be observed in the chromatograms. This is also the case when using gas chromatography columns with different selectivity, or selecting different pairs of ions from the mass spectra of the two compounds. In order for an interference to escape detection, it would have to have the same retention time on different columns and the same mass spectrometric fragmentation pattern, which are very unlikely to occur simultaneously. The accuracy of the procedure is established by the high specificity of the gas chromatography-mass spectrometry technique as reported here and the exact assessment of recovery using the isotope dilution principle.
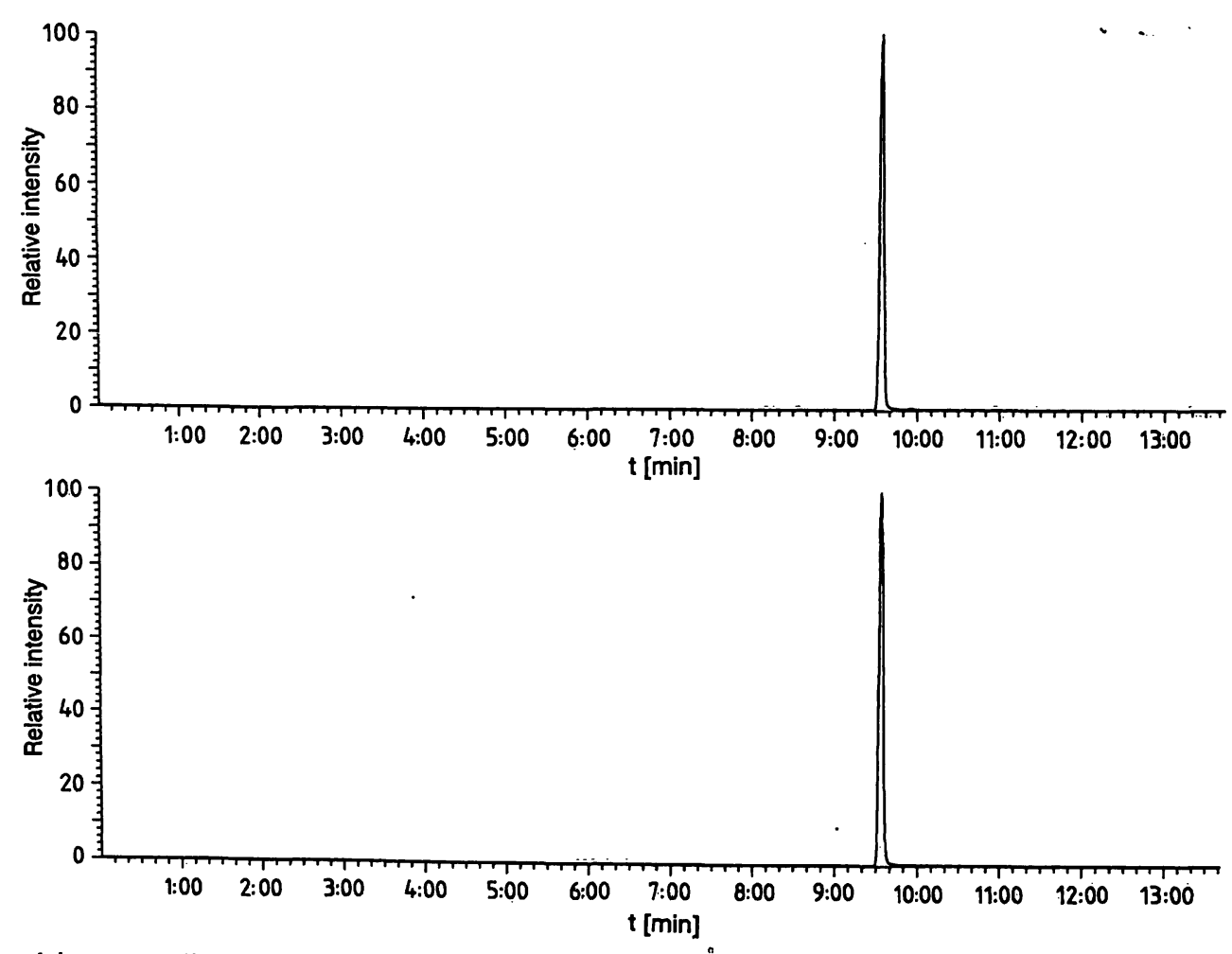

Fig. 1. Selected ion recording of cholesterol trimethylsilyl ether (upper panel, $\mathrm{m} / \mathrm{z} 458$ ) and ${ }^{13} \mathrm{C}_{2}$-cholesterol trimethylsilyl ether (lower panel, $\mathrm{m} / \mathrm{z} 460$ ) after processing a serum sample. 
Similarly, only the glycerol derivative and the corresponding labelled compound are recorded when the selected ion recording method is used for the analysis of total glycerol.

The performance criteria of the reference measurement procedure for cholesterol may be judged from measurement of the control serum 909 of the NIST. The bias with reference to the certified values of the NIST is generally lower than $0.5 \%$ in three different surveys. The imprecision for the cholesterol reference method is in the order of 0.34 to $0.69 \%$ (CV) and for total glycerol in the range from 0.30 to $1.00 \%(\mathrm{CV})$.

\section{Discussion}

It should emphasized that the technique of isotope dilution mass spectrometry is not a reference method in itself; one should keep in mind that markedly biased results can be obtained, despite the fact that a highly specific and accurate analytical technique is used.

The application of an analytical principle such as isotope dilution mass spectrometry is only one of the necessary prerequisites for a reference method. In addition it is necessary to use well characterized primary reference substances of high purity for calibration. The balances and the volumetric equipment used for sampling the sera and measuring the standard solutions must be carefully calibrated. It is worthwhile to note that a detailed protocol must be followed when setting up reference method values. This includes repeated analyses on at least three independent occasions, which means that a new standard solution has to be prepared each time from the certified primary standard material. In order to demonstrate the accuracy of the procedure, control sera, e.g. the NIST serum 909 , must be included in each analytical series. The precision of the results obtained from several different series of measurement must be within an acceptable range.

\section{References}

1. Anonymous (1988) Qualitätssicherung der quantitativen Bestimmungen im Labọratorium. Dt. Ärzteblatt 85, A697A712.

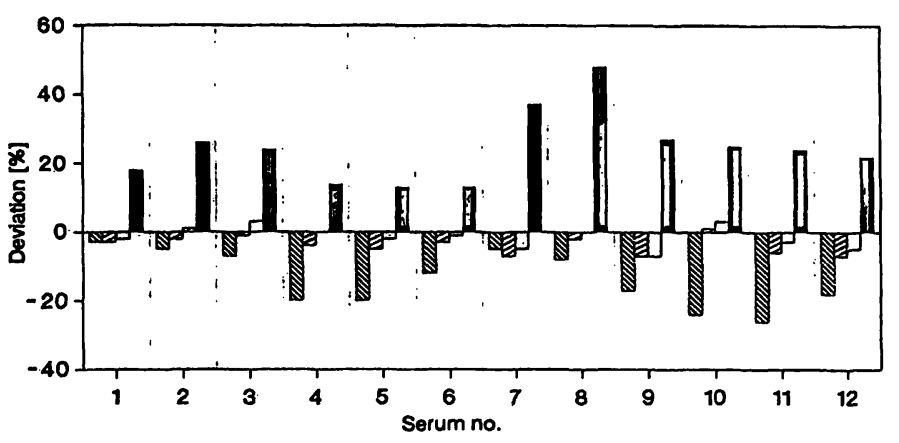

Fig. 2. Deviation of method-dependent target values from isotope dilution mass spectrometry reference method values in control sera for external quality assessment of cholesterol analysis.

WWW cholesterol oxidase-iodide
cholesterol oxidase-p-aminophenazone
cholesterol oxidase-catalase
Liebermann-Burchard

In the external quality assessment scheme used in Germany before 1989, method-dependent target values were set up to evaluate the participants' results. It can be demonstrated from figure 2 that the Liebermann-Burchard reaction gave values that were up to $50 \%$ too high, and the cholesterol oxidase-iodide method gave values up to $20 \%$ too low. Only the cholesterol oxidase-p-aminophenazone and the cholesterol oxidase-catalase method agreed with the isotope dilution mass spectrometry results. This example clearly demonstrates that there was an unacceptably wide scatter of method-dependent target values. Following the introduction of reference methods as a basis for the evaluation of results in external quality assessment in 1989, laboratories have replaced inaccurate methods for lipid analysis e. g. the LiebermannBurchard procedure or the cholesterol oxidase-iodide method by the more accurate cholesterol oxidase- $p$ aminophenazone and cholesterol oxidase-catalase techniques, thereby considerably improving the comparability of lipid analyses in different laboratories.

Prof. Dr. L. Siekmann

Institut für Klinische Biochemie

der Universität Bonn

Sigmund-Freud-Straße 25

W-5300 Bonn 1

Bundesrepublik Deutschland 
